\section{Improving the Productivity, Quality, and Storability of 'Katja' Apple by Better Orchard Management Procedures}

\author{
Ibrahim I. Tahir ${ }^{1}$, Eva Johansson, and Marie E. Olsson \\ Faculty of Landscape Planning, Horticulture and Agricultural Sciences, The \\ Swedish University of Agricultural Sciences, P.O. Box 44, SE-230 53 Alnarp, \\ Sweden
}

Additional index words. Apple (Malus domestica), pruning, bark mulching, fertigation, Pezicula malicorticis

\begin{abstract}
This 4-year study at the Kivik research station, southeastern Sweden $\left(\approx 55^{\circ} \mathrm{N}\right)$, investigated optimal orchard management procedures to improve tree growth, yield, fruit quality, and storability in the important early season apple cultivar 'Katja'. Two procedures (one standard, one novel) were applied within pruning, weed control, fertigation, and thinning, yielding a total of 16 different combinations (treatments). Tree vegetative growth, yield, fruit size, fruit color, firmness, soluble solids concentration, malic acid, nitrogen and calcium content, and percentage fruit with storage rot (Pezicula malicorticis) were evaluated for all 16 treatments. Changing to the novel alternative in only one or two of the areas did not produce significant improvements in all investigated parameters. The optimal treatment consisted of radical winter pruning + summer pruning (novel), bark mulching (novel), fertigation with $0.13 \mathrm{~g} N$ (standard), and hand thinning after full bloom (novel).
\end{abstract}

'Katja' is a commercially important early season apple cultivar in Sweden. Difficulties such as very weak growth, rapid quality deterioration, and low fruit storage potential limit growers' ability to meet quality standards set by markets (http://www.algonet.se). These problems could be alleviated through better understanding of the close relationships between cultural practices and fruit storability (Tromp, 2005).

Pruning intensity, time, and method have varying effects on apple quality at harvest and during storage (Tomala, 1997; Wertheim, 2005). Winter pruning improves tree growth, fruit coloration, acidity, sugar content, and storability and increases fruit weight (Marini and Barden, 2004; Tahir et al., 2007). Summer pruning further improves fruit coloration and size but decreases yield and causes poor fruit storability (Fathi and Mukhtar, 1998; Goldschmidt, 1997; Tromp, 2005). Covering the soil surface in orchards instead of using herbicides can prevent weeds, change soil environmental conditions, improve availability of nutrients to trees, enhance vegetative growth, increase yield, and improve fruit storability (Rubauskis et al., 2002; Tahir et al., 2005). However, in some cases, soil covering can decrease fruit yield and sugar content (Fausett and Rom, 2001). Fertigation systems need careful adjustment, because higher nitrogen levels or

Received for publication 29 Nov. 2007. Accepted for publication 16 Feb. 2008.

${ }^{1}$ To whom reprint requests should be addressed; e-mail ibrahim.tahir@ltj.slu.se excess water supply can cause poor fruit storability (Meheriuk et al., 1996; Rubauskis et al., 2002). Fertilization and irrigation normally increase trunk cross-sectional area and yield (Ghosh et al., 2004; Jadczuk et al., 2001). The international trend for organic cultivation practices rather than chemical has renewed interest in hand thinning (Pretorious et al., 2004). In most cases, thinning increases fruit size (Marini, 2004; Tomala, 1997), firmness, and sweetness (Basak, 1999; Bergh, 1990) and improves superficial color (Link, 2000). Although some studies report no visible effects of thinning on fruit storability (Krzewinska et al., 2002), others report a tendency for decreased storage potential related to thinning (Wójcik et al., 2001) and decreased yield (Basak, 1999).

Most studies relating apple fruit quality to orchard management procedures involve individual testing of each practice. This study sought to improve 'Katja' apple quality and storability by combining different management practices, namely application of bark mulch, pruning, hand thinning, and nitrogen fertigation, regulated at two different levels, resulting in 16 treatments.

\section{Materials and Methods}

Experimental design and treatments

The influence of management procedures on fruit yield and quality was investigated in a 'Katja' apple orchard at Kivik research station, southeastern Sweden $\left(\approx 55^{\circ} \mathrm{N}\right)$, during four growing seasons (2000 to 2003) to eliminate confounding climate effects. Trees were grafted onto M26 rootstock and planted in $1997(4 \mathrm{~m}$ interrow $\times 2 \mathrm{~m}$ intertree spacing). Fruit first appeared only 1 year later, resulting in very weak vegetative growth and poor fruit quality. A completely randomized block design with six single tree blocks was used in the study. All trees were trained as Geneva $\mathrm{Y}$-trellis $\approx 1 \mathrm{~m}$ in height, the $\mathrm{Y}$ angle was $60^{\circ}$, and the $\mathrm{Y}$ junction was $0.6 \mathrm{~m}$ above the ground. The investigated orchard management procedures were:

1. Pruning $(\mathrm{P})$. Two types of pruning were tested: a) standard pruning $\left(\mathrm{P}_{\mathrm{s}}\right)$ : all upper shoots growing close to the arms removed and all side shoots shortened to $\approx 30 \mathrm{~cm}$ long in early April. Both arms shortened with a head cut into the basal fifth leaf; and b) radical pruning $\left(\mathrm{P}_{\mathrm{r}}\right)$ : two-thirds of each tree arm pruned and all upper and lateral shoots totally removed or shortened to 5 to $10 \mathrm{~cm}$ in early April of each year. Starting in Year 2, all shoots shortened (weaker removed) again in July to the nearest bearing shoot. All nonproductive limbs causing too much shading thinned and all new shoots arising in the main stem or $\mathrm{Y}$ arms removed. Some drooping shoots head cut to restore horizontal shape.

2. Weed control (W). Soil surface kept free from weeds by: a) herbicides $\left(\mathrm{W}_{\mathrm{s}}\right)$ : mixture of glyphosate (360 g. $\left.\mathrm{L}^{-1}\right)$ Roundup (N-phosphomethylglycine; Monsanto, Brussels, Belgium) and MCPA [Duplosan super, (4-chloro-2methylphenoxy), BASF, Gothenburg, Sweden] sprayed onto the soil twice a year (May, July). Herbicide dose 3 $\mathrm{L} \cdot \mathrm{ha}^{-1}$ on sprayed areas, liquid rate $320 \mathrm{~L} \cdot \mathrm{ha}^{-1}$, and travel speed $3.5 \mathrm{~km} \cdot \mathrm{h}^{-1}$; b) bark mulching $\left(\mathrm{W}_{\mathrm{m}}\right)$ : soil covered during the vegetative period with a layer (20 cm thick) of 1-year-old chopped pine bark. More bark added every year.

3. Fertilization (F). All trees drip-irrigated $(0.8 \mathrm{~L} /$ tree) May to August every season and fertigated as: a) standard fertigation $\left(\mathrm{F}_{\mathrm{s}}\right)$ of $0.13 \mathrm{~g}$ Red Superba and Gartnersalpeter, 1:200 (7N-4P$21 \mathrm{~K}$ with $\mathrm{Mg}, \mathrm{S}$, and micronutrients; HYDROAGRI, Arsta, Sweden); b) excess nitrogen fertigation $\left(\mathrm{F}_{\mathrm{x}}\right)$ with trees given additional nitrogen $[2 \times 25$ $\mathrm{g}(15.5 \% \mathrm{~N}$ and $19.0 \% \mathrm{Ca})$; HYDROAGRI], applied 1 May and 1 June.

4. Thinning (T): a) unthinned trees $\left(\mathrm{T}_{\mathrm{s}}\right)$; b) hand thinning $\left(T_{n}\right)$, all flowers within each cluster except the king flower removed 1 to 2 weeks after full bloom.

These procedures were arranged in 16 different treatments: Tr1: control, standard procedures $\left(\mathrm{P}_{\mathrm{s}} \cdot \mathrm{W}_{\mathrm{s}} \cdot \mathrm{F}_{\mathrm{s}} \cdot \mathrm{T}_{\mathrm{s}}\right)$; $\mathrm{Tr} 2$ to $\operatorname{Tr} 5$ : treatments with one procedure changed compared with control (Tr2: $\mathrm{P}_{\mathrm{r}} \cdot \mathrm{W}_{\mathrm{s}} \cdot \mathrm{F}_{\mathrm{s}} \cdot \mathrm{T}_{\mathrm{s}}$; Tr3: $\mathrm{P}_{\mathrm{s}} \cdot \mathrm{W}_{\mathrm{m}}$. $\left.\mathrm{F}_{\mathrm{s}} \cdot \mathrm{T}_{\mathrm{s}} ; \operatorname{Tr} 4: \mathrm{P}_{\mathrm{s}} \cdot \mathrm{W}_{\mathrm{s}} \cdot \mathrm{F}_{\mathrm{x}} \cdot \mathrm{T}_{\mathrm{s}} ; \operatorname{Tr} 5: \mathrm{P}_{\mathrm{s}} \cdot \mathrm{W}_{\mathrm{s}} \cdot \mathrm{F}_{\mathrm{s}} \cdot \mathrm{T}_{\mathrm{n}}\right)$. Tr6 to Tr11: treatments with two procedures changed compared with control (Tr6: $\mathrm{P}_{\mathrm{r}} \cdot \mathrm{W}_{\mathrm{m}}$. $\mathrm{F}_{\mathrm{s}} \cdot \mathrm{T}_{\mathrm{s}} ; \operatorname{Tr} 7: \mathrm{P}_{\mathrm{r}} \cdot \mathrm{W}_{\mathrm{s}} \cdot \mathrm{F}_{\mathrm{x}} \cdot \mathrm{T}_{\mathrm{s}} ; \operatorname{Tr} 8: \mathrm{P}_{\mathrm{r}} \cdot \mathrm{W}_{\mathrm{s}} \cdot \mathrm{F}_{\mathrm{s}} \cdot \mathrm{T}_{\mathrm{n}}$; Tr9: $\mathrm{P}_{\mathrm{s}} \cdot \mathrm{W}_{\mathrm{m}} \cdot \mathrm{F}_{\mathrm{x}} \cdot \mathrm{T}_{\mathrm{s}}, \operatorname{Tr} 10: \mathrm{P}_{\mathrm{s}} \cdot \mathrm{W}_{\mathrm{m}} \cdot \mathrm{F}_{\mathrm{s}} \cdot \mathrm{T}_{\mathrm{n}} ; \operatorname{Tr} 11$ : 
$\left.\mathrm{P}_{\mathrm{s}} \cdot \mathrm{W}_{\mathrm{s}} \cdot \mathrm{F}_{\mathrm{x}} \cdot \mathrm{T}_{\mathrm{n}}\right) \cdot \operatorname{Tr} 12$ to $\operatorname{Tr} 15$ : treatments with three procedures changed compared with control (Tr12: $\mathrm{P}_{\mathrm{r}} \cdot \mathrm{W}_{\mathrm{m}} \cdot \mathrm{F}_{\mathrm{x}} \cdot \mathrm{T}_{\mathrm{s}} ; \operatorname{Tr} 13: \mathrm{P}_{\mathrm{r}} \cdot \mathrm{W}_{\mathrm{m}} \cdot \mathrm{F}_{\mathrm{s}} \cdot \mathrm{T}_{\mathrm{n}}$; Tr14: $\left.\quad \mathrm{P}_{\mathrm{r}} \cdot \mathrm{W}_{\mathrm{s}} \cdot \mathrm{F}_{\mathrm{x}} \cdot \mathrm{T}_{\mathrm{n}} ; \quad \operatorname{Tr} 15: \quad \mathrm{P}_{\mathrm{s}} \cdot \mathrm{W}_{\mathrm{m}} \cdot \mathrm{F}_{\mathrm{x}} \cdot \mathrm{T}_{\mathrm{n}}\right)$. Tr16: Treatment with all procedures changed compared with control, $\mathrm{P}_{\mathrm{r}} \cdot \mathrm{W}_{\mathrm{m}} \cdot \mathrm{F}_{\mathrm{x}} \cdot \mathrm{T}_{\mathrm{n}}$.

\section{Evaluation}

Tree growth and light distribution. Trunk diameter was measured $30 \mathrm{~cm}$ above the scion-rootstock union. The average of two readings (taken across and along the row) was converted to trunk cross-sectional area (TCSA; 3.14 stem radius $^{2}$ ). Total shoot length was measured in May (before summer pruning) of each year. Light distribution within the crown was measured by light meter (L1-188; L1-COR, Frederickson, Copenhagen, Denmark). Data were recorded at the center of the trunk from the four directions of the crown at $70 \mathrm{~cm}$ above the ground. Light $\left(\mu \mathrm{Em}^{2} \cdot \mathrm{sec}^{-1}\right)$ was estimated on cloudless days on three occasions during 2001 at two positions per procedure. Full sunlight was measured (same time and height) in a free area (between rows). Data are presented as mean percentage transmission.

Fruit quality. Optimum harvesting date was determined by estimating starch score three times a week during the maturation period. Fruit firmness was estimated with a penetrometer (model FT 327; Effigy, Milan, Italy; plunger diameter $11.1 \mathrm{~mm}$ ) on opposite sides of each fruit. Subsamples were homog- enized in a Waring blender ( $1 \mathrm{~min})$ with ultrapure water $(1: 1 ; \mathrm{w} / \mathrm{v})$. After centrifugation $(10,000 \times \mathrm{g})$ for $10 \mathrm{~min}$, percentage soluble solids concentration (SSC) was determined with a refractrometer (RMF 80; Bellingham and Standley Ltd., UK). From the supernatant, $5 \mathrm{~mL}$ were diluted with $20 \mathrm{~mL}$ ultrapure water and titrated as malic acid with $\mathrm{NaOH}(0.05 \mathrm{M})$ to $\mathrm{pH} 8.1$ with a Radiometer (PHM64; Denmark). Fruit surface color was assessed with a Chroma Meter model CR 200 with an 8-mm diameter window (Minolta Camera Co., Ltd., Osaka, Japan), at three spots per fruit and expressed as $\mathrm{H}^{\circ}$, i.e., hue angle $\left(\mathrm{H}^{\circ}=\tan ^{-1} \mathrm{~b}^{*} / \mathrm{a} *\right)$, where $\mathrm{b}^{*}$ is the yellow/blue and $\mathrm{a}^{*}$ the red/green color coordinates. $\mathrm{H}^{\circ}=0$, totally red, $\mathrm{H}^{\circ}=90^{\circ}$ totally yellow and $\mathrm{H}^{\circ}=180^{\circ}$ totally green peel color (McGuire, 1992). Two additional fruits from each tree were analyzed at harvesting to estimate the mineral content $(\mathrm{N}, \mathrm{K}, \mathrm{Ca}$, and $\mathrm{Mg}$ ), using NMKL6 and $\mathrm{HNO}_{3}$ - ICP-AES methods.

Fruit storability. Samples of 30 fruits from each block/treatment were stored at $2.5{ }^{\circ} \mathrm{C}$ and $90 \%$ relative humidity for 10 weeks. Fruit quality was estimated after storage as explained previously. The percentage incidence of storage decay was visually determined according to the storage decay index described in Rein (1996).

Statistical analyses. For light transmission, yield, and fruit weight of 'Katja' apples, means and SDs were calculated using the MS Excel program (Microsoft, Redmond, WA).
Following recommendations in Fernandez (2007), analysis of variance (ANOVA procedure) was carried out using SAS (SAS Inst. Inc., Cary, NC). Four comparisons of various managements and their interactions on tree vegetative growth, trunk growth, yield, and fruit quality parameters were analyzed. After ANOVA analyses, the Turkey's studentized range at $P<0.05$ was calculated for each of the parameters in the 16 treatments.

\section{Results}

Vegetative growth and light transmission. Over the 4 years, standard orchard management $\left(\mathrm{P}_{\mathrm{s}} \cdot \mathrm{W}_{\mathrm{s}} \cdot \mathrm{F}_{\mathrm{s}} \cdot \mathrm{T}_{\mathrm{s}}\right)$ of 'Katja' apples caused slow growth (27\% relative increase in TCSA) and weak trees (total annual new growth of shoots was $18 \mathrm{~cm}$ ) (Tables 1 and $2 ; P<0.05$ ). Radical pruning $\left(\mathrm{P}_{\mathrm{r}} \cdot \mathrm{W}_{\mathrm{s}} \cdot \mathrm{F}_{\mathrm{s}} \cdot \mathrm{T}_{\mathrm{s}}\right)$ increased TCSA (by 38\%) and produced three times longer new shoots than the control trees $\left(\mathrm{P}_{\mathrm{s}} \cdot \mathrm{W}_{\mathrm{s}} \cdot \mathrm{F}_{\mathrm{s}} \cdot \mathrm{T}_{\mathrm{s}}\right)$. Bark mulching $\left(\mathrm{P}_{\mathrm{s}} \cdot \mathrm{W}_{\mathrm{m}} \cdot \mathrm{F}_{\mathrm{s}} \cdot \mathrm{T}_{\mathrm{s}}\right)$, application of excess $\mathrm{N}$ fertigation $\left(\mathrm{P}_{\mathrm{s}} \cdot \mathrm{W}_{\mathrm{s}} \cdot \mathrm{F}_{\mathrm{x}} \cdot \mathrm{T}_{\mathrm{s}}\right)$ or hand thinning $\left(\mathrm{P}_{\mathrm{s}} \cdot \mathrm{W}_{\mathrm{s}} \cdot \mathrm{F}_{\mathrm{s}} \cdot \mathrm{T}_{\mathrm{n}}\right)$ had no significant effect on trunk growth and annual shoot growth in comparison with control trees (Table 2).

Although changing two procedures of four (Tr. 6 to 11) slightly improved vegetative growth compared with standard or individual procedures (Tr. 1 to 5), the effects were lower than for radical pruning alone (Tables 1 and 2). Of the treatments with three

Table 1. Mean squares from analyses of variance of shoot length, yield, fruit weight and fruit quality, and storability as related to orchard management. ${ }^{z}$

\begin{tabular}{|c|c|c|c|c|c|c|c|c|c|}
\hline Procedures & df & $\begin{array}{l}\text { Shoot } \\
\left(10^{-3}\right)\end{array}$ & $\begin{array}{l}\text { Yield } \\
\left(10^{-3}\right)\end{array}$ & $\begin{array}{c}\text { Fruit wt } \\
\left(10^{-3}\right)\end{array}$ & $\begin{array}{l}\text { Color } \\
\left(10^{-3}\right)\end{array}$ & Firmness & SSC & $\begin{array}{c}\text { Malic } \\
\text { acid }\end{array}$ & $\begin{array}{c}\text { Decay } \\
\left(1^{-1}\right)\end{array}$ \\
\hline$\overline{\mathrm{P}}$ & 1 & $125.0 * * *$ & $5.3 * * *$ & $20.4^{* * *}$ & 0.4 & $54.9 * * *$ & $6.0 * * *$ & 0.0 & 0.1 \\
\hline W & 1 & $35.1 * * *$ & $3.8 * * *$ & $19.0 * * *$ & $2.8 * * *$ & $28.0 * * *$ & 1.1 & $7.9 * * *$ & 0.7 \\
\hline $\mathrm{F}$ & 1 & $8.9 * * *$ & $7.5 * * *$ & $4.6^{* * *}$ & $2.9 * * *$ & $67.0 * * *$ & $5.8 * * *$ & $8.5 * * *$ & 1.4 \\
\hline $\mathrm{T}$ & 1 & $15.0 * * *$ & $5.8 * * *$ & $12.8 * * *$ & $3.8 * * *$ & $9.5^{* * *}$ & 0.0 & $7.5 * * *$ & $4.1^{*}$ \\
\hline $\mathrm{Y}$ & 3 & $13.9 * * *$ & 0.1 & $1.9 * * *$ & $1.4 * * *$ & $36.7 * * *$ & $20.0 * * *$ & $19.3 * * *$ & 1.8 \\
\hline $\mathrm{P} * \mathrm{~W}$ & 1 & $23.0 * * *$ & $6.1 * * *$ & 0.2 & $3.9 * * *$ & $4.8 * * *$ & 0.5 & 0.3 & 0.0 \\
\hline $\mathrm{P} * \mathrm{~F}$ & 1 & $5.3 * * *$ & $5.4 * * *$ & 0.0 & $4.8 * * *$ & 0.1 & 0.0 & 0.1 & 0.0 \\
\hline $\mathrm{P} * \mathrm{~T}$ & 1 & $14.0 * * *$ & $5.0 * * *$ & $4.9 * * *$ & $2.6 * * *$ & 1.1 & 0.0 & 0.3 & 0.4 \\
\hline $\mathrm{P}^{*} \mathrm{Y}$ & 3 & $1.1 *$ & 0.0 & $0.4^{*}$ & $0.4 * * *$ & 0.3 & $2.2 * * *$ & 0.5 & 0.7 \\
\hline $\mathrm{W} * \mathrm{~F}$ & 1 & $3.6 * * *$ & $5.9 * * *$ & 0.0 & $6.5^{* * *}$ & 0.2 & 0.0 & $1.2 *$ & 3.2 \\
\hline $\mathrm{W}^{*} \mathrm{~T}$ & 1 & $18.0 * * *$ & $5.2 * * *$ & 0.5 & $1.6 * * *$ & $4.6^{* * *}$ & 1.3 & $1.0^{*}$ & 0.3 \\
\hline $\mathrm{W}^{*} \mathrm{Y}$ & 3 & $1.5 * * *$ & 0.0 & 0.0 & 0.2 & 0.5 & 1.3 & $0.8^{*}$ & 1.1 \\
\hline $\mathrm{F}^{*} \mathrm{~T}$ & 1 & 0.0 & $5.9 * * *$ & $3.1 * * *$ & $0.6 * * *$ & $1.5^{*}$ & 0.7 & $7.1 * * *$ & 2.8 \\
\hline $\mathrm{F}^{*} \mathrm{Y}$ & 3 & 0.5 & 0.0 & 0.2 & 0.0 & $2.7 * * *$ & $1.5^{*}$ & 0.5 & 0.5 \\
\hline $\mathrm{T}^{*} \mathrm{Y}$ & 3 & $0.9 *$ & 0.0 & 0.3 & $0.2^{*}$ & $4.1 * * *$ & 1.0 & $2.5 * * *$ & $2.2 *$ \\
\hline $\mathrm{P} * \mathrm{~W}^{*} \mathrm{~T}$ & 1 & $12.4 * * *$ & $5.3 * * *$ & $8.5 * * *$ & $2.5 * * *$ & $20.2 * * *$ & 0.0 & 0.1 & 0.8 \\
\hline $\mathrm{P} * \mathrm{~W} * \mathrm{~F}$ & 1 & 0.0 & $5.5 * * *$ & $5.1 * * *$ & $3.9 * * *$ & $23.9 * * *$ & $3.2 *$ & 0.0 & 0.5 \\
\hline $\mathrm{P}^{*} \mathrm{~W}^{*} \mathrm{Y}$ & 3 & $0.5^{*}$ & 0.0 & 0.3 & $0.3^{* * *}$ & 0.0 & 0.9 & 0.1 & 0.4 \\
\hline $\mathrm{P} * \mathrm{~F}^{*} \mathrm{~T}$ & 1 & 1.0 & $5.6 * * *$ & $1.0^{*}$ & $3.0 * * *$ & $1.4^{*}$ & $2.1^{*}$ & 0.1 & 1.0 \\
\hline $\mathrm{P} * \mathrm{~T} * \mathrm{Y}$ & 3 & $1.3 * *$ & 0.0 & 0.4 & $0.3 * * *$ & $1.6^{* * *}$ & 0.4 & 0.2 & 1.6 \\
\hline $\mathrm{P}^{*} \mathrm{~F}^{*} \mathrm{Y}$ & 3 & 0.2 & 0.0 & $0.5^{*}$ & $0.5 * * *$ & 0.6 & 0.2 & 0.2 & 0.6 \\
\hline $\mathrm{W} * \mathrm{~F}^{*} \mathrm{~T}$ & 1 & 0.2 & $6.1 * * *$ & 0.5 & $3.1 * * *$ & 0.6 & $3.6^{* *}$ & 0.0 & 0.3 \\
\hline $\mathrm{W}^{*} \mathrm{~F}^{*} \mathrm{Y}$ & 3 & $2.0 * * *$ & 0.0 & 0.2 & $0.3^{* * *}$ & $3.3 * * *$ & 0.9 & $0.9 * *$ & 1.6 \\
\hline $\mathrm{W}^{*} \mathrm{~T}^{*} \mathrm{Y}$ & 3 & $1.9 * * *$ & 0.0 & 0.0 & 0.0 & $1.0^{*}$ & 0.4 & $3.5 * * *$ & 0.6 \\
\hline $\mathrm{F}^{*} \mathrm{~T} * \mathrm{Y}$ & 3 & $1.0 *$ & 0.0 & 0.0 & 0.2 & 0.7 & $2.2 * *$ & 0.7 & 0.2 \\
\hline $\mathrm{P} * \mathrm{~W}^{*} \mathrm{~F}^{*} \mathrm{~T}$ & 1 & $7.3 * * *$ & $5.7 * * *$ & $2.5 * * *$ & $3.5 * * *$ & $41.3^{* * *}$ & 1.6 & 0.1 & 1.3 \\
\hline $\mathrm{P}^{*} \mathrm{~W}^{*} \mathrm{~F}^{*} \mathrm{Y}$ & 3 & 0.8 & 0.0 & 0.0 & 0.1 & 0.6 & 0.6 & 0.4 & 0.5 \\
\hline $\mathrm{P} * \mathrm{~F}^{*} \mathrm{~T} * \mathrm{Y}$ & 3 & 0.8 & 0.0 & 0.0 & $0.2^{*}$ & 1.6 & 1.2 & 0.4 & 0.7 \\
\hline $\mathrm{P} * \mathrm{~W}^{*} \mathrm{~T} * \mathrm{Y}$ & 3 & $1.2 *$ & 0.0 & 0.3 & 0.0 & 0.5 & 0.6 & 0.3 & 0.5 \\
\hline $\mathrm{W}^{*} \mathrm{~F}^{*} \mathrm{~T}^{*} \mathrm{Y}$ & 3 & $1.0^{*}$ & 0.0 & $1.1 * * *$ & 0.0 & 1.0 & 1.1 & 0.5 & 1.4 \\
\hline $\mathrm{P} * \mathrm{~W}^{*} \mathrm{~F}^{*} \mathrm{~T} * \mathrm{Y}$ & 3 & 0.6 & 0.0 & 0.1 & $0.3 * *$ & 2.4 & 1.2 & 0.0 & 0.5 \\
\hline Error & 128 & 40.0 & 3.6 & $20.6^{* * *}$ & 8.3 & 42.5 & 64.8 & 27.8 & 105.2 \\
\hline
\end{tabular}

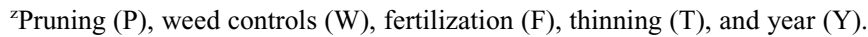

${ }^{*},{ }^{* *},{ }^{* * *}$ Significant at $P<0.5,0.1,0.05$, respectively.

$\mathrm{SSC}=$ soluble solids concentration. 
Table 2. Vegetative growth, tree productivity and fruit quality of Katja apple resulting from application of different management procedures (2000 to 2003 average). ${ }^{z}$

\begin{tabular}{|c|c|c|c|c|c|c|c|}
\hline Tr. & Procedures & $\begin{array}{c}\text { TCSA }^{\mathrm{y}} \\
\text { Relative } \\
\text { change }(\%)\end{array}$ & $\begin{array}{c}\text { Total } \\
\text { annual } \\
\text { new shoots } \\
(\mathrm{cm})\end{array}$ & $\begin{array}{l}\text { Color } \\
\left(\mathrm{H}^{\circ}\right)\end{array}$ & $\begin{array}{c}\text { Firmness } \\
(\mathrm{N})\end{array}$ & $\begin{array}{c}\mathrm{SSC}^{\mathrm{x}} \\
(\%)\end{array}$ & $\begin{array}{c}\text { Malic acid } \\
\left(\mathrm{mg} / 100 \mathrm{~g}^{-1}\right. \\
\text { FW })\end{array}$ \\
\hline 1 & $\mathrm{P}_{\mathrm{s}} \cdot \mathrm{W}_{\mathrm{s}} \cdot \mathrm{F}_{\mathrm{s}} \cdot \mathrm{T}_{\mathrm{s}}$ & $26.8 \mathrm{~d}$ & $17.4 \mathrm{e}$ & $70.7 \mathrm{a}$ & $70.1 \mathrm{f}$ & $11.6 \mathrm{a}$ & $664.0 \mathrm{~cd}$ \\
\hline 2 & $\mathrm{P}_{\mathrm{r}} \cdot \mathrm{W}_{\mathrm{s}} \cdot \mathrm{F}_{\mathrm{s}} \cdot \mathrm{T}_{\mathrm{s}}$ & $37.0 \mathrm{c}$ & $53.0 \mathrm{bcd}$ & 52.3 bcde & 77.7 cde & $10.8 \mathrm{a}$ & $688.6 \mathrm{bcd}$ \\
\hline 3 & $\mathrm{P}_{\mathrm{s}} \cdot \mathrm{W}_{\mathrm{m}} \cdot \mathrm{F}_{\mathrm{s}} \cdot \mathrm{T}_{\mathrm{s}}$ & $29.5 \mathrm{~d}$ & $25.8 \mathrm{de}$ & 57.3 abcde & $80.6 \mathrm{abc}$ & $11.5 \mathrm{a}$ & $656.8 \mathrm{~cd}$ \\
\hline 4 & $P_{s} \cdot W_{s} \cdot F_{x} \cdot T_{s}$ & $30.8 \mathrm{~d}$ & $30.6 \mathrm{de}$ & $62.8 \mathrm{abcd}$ & $74.1 \mathrm{e}$ & $11.7 \mathrm{a}$ & $706.3 \mathrm{bc}$ \\
\hline 5 & $\mathrm{P}_{\mathrm{s}} \cdot \mathrm{W}_{\mathrm{s}} \cdot \mathrm{F}_{\mathrm{s}} \cdot \mathrm{T}_{\mathrm{n}}$ & $35.4 \mathrm{~d}$ & $32.4 \mathrm{de}$ & 56.3 abcde & $75.9 \mathrm{de}$ & $11.5 \mathrm{a}$ & $673.6 \mathrm{~cd}$ \\
\hline 6 & $\mathrm{P}_{\mathrm{r}} \cdot \mathrm{W}_{\mathrm{m}} \cdot \mathrm{F}_{\mathrm{s}} \cdot \mathrm{T}_{\mathrm{s}}$ & $50.3 \mathrm{c}$ & 46.2 cde & $49.2 \mathrm{cde}$ & $84.0 \mathrm{ab}$ & $12.6 \mathrm{a}$ & $895.8 \mathrm{a}$ \\
\hline 7 & $\mathrm{P}_{\mathrm{r}} \cdot \mathrm{W}_{\mathrm{s}} \cdot \mathrm{F}_{\mathrm{x}} \cdot \mathrm{T}_{\mathrm{s}}$ & $43.1 \mathrm{c}$ & 51.3 bcde & $62.6 \mathrm{abcd}$ & $75.1 \mathrm{e}$ & $11.6 \mathrm{a}$ & $703.4 \mathrm{bcd}$ \\
\hline 8 & $P_{r} \cdot W_{s} \cdot F_{s} \cdot T_{n}$ & $52.6 \mathrm{c}$ & 30.2 cde & $47.3 \mathrm{de}$ & $80.8 \mathrm{abc}$ & $11.7 \mathrm{a}$ & $671.1 \mathrm{~cd}$ \\
\hline 9 & $\mathrm{P}_{\mathrm{s}} \cdot \mathrm{W}_{\mathrm{m}} \cdot \mathrm{F}_{\mathrm{x}} \cdot \mathrm{T}_{\mathrm{s}}$ & $42.8 \mathrm{c}$ & $25.9 \mathrm{de}$ & 57.7 abcde & $74.1 \mathrm{e}$ & $12.2 \mathrm{a}$ & $648.2 \mathrm{~d}$ \\
\hline 10 & $\mathrm{P}_{\mathrm{s}} \cdot \mathrm{W}_{\mathrm{m}} \cdot \mathrm{F}_{\mathrm{s}} \cdot \mathrm{T}_{\mathrm{n}}$ & $43.1 \mathrm{c}$ & $19.1 \mathrm{de}$ & 48.9 cde & $81.7 \mathrm{abc}$ & $11.6 \mathrm{a}$ & $839.2 \mathrm{a}$ \\
\hline 11 & $\mathrm{P}_{\mathrm{s}} \cdot \mathrm{W}_{\mathrm{s}} \cdot \mathrm{F}_{\mathrm{x}} \cdot \mathrm{T}_{\mathrm{n}}$ & $42.5 \mathrm{c}$ & $24.5 \mathrm{de}$ & $69.3 \mathrm{ab}$ & $79.5 \mathrm{~cd}$ & $12.1 \mathrm{a}$ & $742.4 \mathrm{~b}$ \\
\hline 12 & $\mathrm{P}_{\mathrm{r}} \cdot \mathrm{W}_{\mathrm{m}} \cdot \mathrm{F}_{\mathrm{x}} \cdot \mathrm{T}_{\mathrm{s}}$ & $62.1 \mathrm{~b}$ & $85.1 \mathrm{~b}$ & $65.7 \mathrm{abc}$ & $76.4 \mathrm{de}$ & $12.7 \mathrm{a}$ & $692.8 \mathrm{bcd}$ \\
\hline 13 & $\mathrm{P}_{\mathrm{r}} \cdot \mathrm{W}_{\mathrm{m}} \cdot \mathrm{F}_{\mathrm{s}} \cdot \mathrm{T}_{\mathrm{n}}$ & $71.2 \mathrm{a}$ & $121.4 \mathrm{a}$ & $42.5 \mathrm{e}$ & $85.0 \mathrm{a}$ & $11.3 \mathrm{a}$ & $657.9 \mathrm{~cd}$ \\
\hline 14 & $\mathrm{P}_{\mathrm{r}} \cdot \mathrm{W}_{\mathrm{s}} \cdot \mathrm{F}_{\mathrm{x}} \cdot \mathrm{T}_{\mathrm{n}}$ & $55.4 \mathrm{ab}$ & $66.7 \mathrm{bc}$ & $67.2 \mathrm{ab}$ & $76.6 \mathrm{de}$ & $11.4 \mathrm{a}$ & $696.2 \mathrm{bcd}$ \\
\hline 15 & $\mathrm{P}_{\mathrm{s}} \cdot \mathrm{W}_{\mathrm{m}} \cdot \mathrm{F}_{\mathrm{x}} \cdot \mathrm{T}_{\mathrm{n}}$ & $64.6 \mathrm{ab}$ & 40.5 cde & $72.4 \mathrm{a}$ & $74.8 \mathrm{e}$ & $11.7 \mathrm{a}$ & $683.9 \mathrm{~cd}$ \\
\hline 16 & $\mathrm{P}_{\mathrm{r}} \cdot \mathrm{W}_{\mathrm{m}} \cdot \mathrm{F}_{\mathrm{x}} \cdot \mathrm{T}_{\mathrm{n}}$ & $72.2 \mathrm{a}$ & $150.3 \mathrm{a}$ & $66.5 \mathrm{ab}$ & $77.5 \mathrm{de}$ & $11.2 \mathrm{a}$ & $668.8 \mathrm{~cd}$ \\
\hline
\end{tabular}

Means in the same column followed by same letter did not differ significantly at $P<0.05$ (Turkey's studentized range- - honestly significant difference).

${ }^{\text {zPruning }}(\mathrm{P})$ - standard $\left(\mathrm{P}_{\mathrm{s}}\right)$, radical $\left(\mathrm{P}_{\mathrm{r}}\right)$; weed control $(\mathrm{W})$ - herbicides $\left(\mathrm{W}_{\mathrm{s}}\right)$, bark mulching $\left(\mathrm{W}_{\mathrm{m}}\right)$; fertilization $(\mathrm{F})$ - standard fertigation $\left(\mathrm{F}_{\mathrm{s}}\right)$, excess nitrogen $\left(\mathrm{F}_{\mathrm{x}}\right)$; and thinning $(\mathrm{T})$ - no thinning $\left(\mathrm{T}_{\mathrm{s}}\right)$, hand thinning $\left(\mathrm{T}_{\mathrm{n}}\right)$.

${ }^{\mathrm{y}}$ Trunk cross-sectional area $(\mathrm{n}=96)$.

${ }^{\mathrm{x}}$ Soluble solid concentration. $\mathrm{H}^{\circ}=\left[\tan ^{-1}\left(\mathrm{~b}^{*} \cdot \mathrm{a}^{-1^{*}}\right)\right]$ where $\mathrm{b}^{*}$ is the yellow-blue and $\mathrm{a}^{*}$ is the red-green color coordinates. $\mathrm{H}^{\circ}=0$ represents totally red, $\mathrm{H}^{\circ}=90^{\circ}$ represents totally yellow, and $\mathrm{H}^{\circ}=180^{\circ}$ represents a totally green peel color.

$\mathrm{FW}=$ fresh weight.

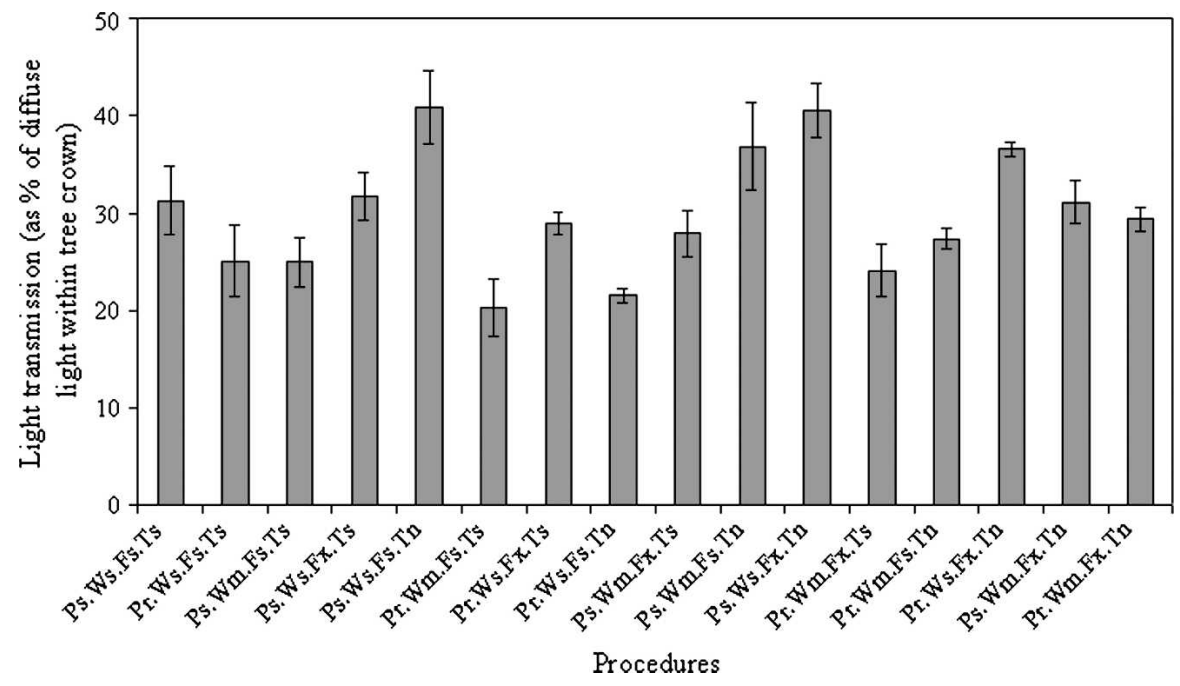

Fig. 1. Light transmission as percent of diffuse light within tree crown of 'Katja' apple trees in July to Aug. 2001. Bars represent $S D \pm(n=96)$. Data were obtained by dividing the within canopy photosynthetic photon flux $(P P F)$ by the simultaneously recorded open sky $P P F$. Procedures: Pruning $(\mathrm{P})$ - standard $\left(\mathrm{P}_{\mathrm{s}}\right)$ or radical $\left(\mathrm{P}_{\mathrm{r}}\right)$; weed control $(\mathrm{W})$ - herbicides $\left(\mathrm{W}_{\mathrm{s}}\right)$ or bark mulching $\left(\mathrm{W}_{\mathrm{m}}\right)$; fertilization (F) - standard fertigation $\left(\mathrm{F}_{\mathrm{s}}\right)$ or excess nitrogen $\left(\mathrm{F}_{\mathrm{x}}\right)$; and thinning $(\mathrm{T})$ - no thinning $\left(\mathrm{T}_{\mathrm{s}}\right)$ or hand thinning $\left(\mathrm{T}_{\mathrm{n}}\right)$.

novel procedures (Tr. 12 to 15$), \mathrm{P}_{\mathrm{r}} \cdot \mathrm{W}_{\mathrm{m}} \cdot \mathrm{F}_{\mathrm{s}} \cdot \mathrm{T}_{\mathrm{n}}$ caused the highest relative increase in TCSA ( $165 \%$ in comparison with standard managed and $92 \%$ in comparison with radically pruned trees) (Tables 1 and 2). Total annual new growth of shoots in trees that were managed by these procedures was six times longer than that in control trees and two times longer than in radically pruned trees (Tables 1 and 2). Even larger trunk and higher increase in shoots growth were achieved with Tr.16 $\left(\mathrm{P}_{\mathrm{r}} \cdot \mathrm{W}_{\mathrm{m}} \cdot \mathrm{F}_{\mathrm{x}} \cdot \mathrm{T}_{\mathrm{n}}\right)$, where trunks were $170 \%$ thicker than trunks of control trees and 95\% thicker
Excess $\mathrm{N}$ fertigation $\left(\mathrm{P}_{\mathrm{s}} \cdot \mathrm{W}_{\mathrm{s}} \cdot \mathrm{F}_{\mathrm{x}} \cdot \mathrm{T}_{\mathrm{s}}\right)$ decreased the incoming light measured at $70 \mathrm{~cm}$ by $20 \%$ in comparison with control trees (Fig. $1 ; P<0.05$ ). $\mathrm{P}_{\mathrm{s}} \cdot \mathrm{W}_{\mathrm{m}} \cdot \mathrm{F}_{\mathrm{x}} \cdot \mathrm{T}_{\mathrm{s}}$ or $\mathrm{P}_{\mathrm{s}} \cdot \mathrm{W}_{\mathrm{s}}$. $\mathrm{F}_{\mathrm{x}} \cdot \mathrm{T}_{\mathrm{n}}$ caused the same negative effect. A three-procedure treatment, $\mathrm{P}_{\mathrm{s}} \cdot \mathrm{W}_{\mathrm{m}} \cdot \mathrm{F}_{\mathrm{x}} \cdot \mathrm{T}_{\mathrm{n}}$, also showed $15 \%$ less light transmission (Fig. 1), whereas $\mathrm{P}_{\mathrm{r}} \cdot \mathrm{W}_{\mathrm{s}} \cdot \mathrm{F}_{\mathrm{s}} \cdot \mathrm{T}_{\mathrm{s}}$ or $\mathrm{P}_{\mathrm{r}} \cdot \mathrm{W}_{\mathrm{m}} \cdot \mathrm{F}_{\mathrm{s}} \cdot \mathrm{T}_{\mathrm{n}}$ improved light transmission by $39 \%$ to $45 \%$ in comparison with standard treatment. It also decreased the negative effect of other practices on the light penetration within the crown when these practices were applied in a fourfactor treatment $\left(\mathrm{P}_{\mathrm{r}} \cdot \mathrm{W}_{\mathrm{m}} \cdot \mathrm{F}_{\mathrm{x}} \cdot \mathrm{T}_{\mathrm{n}}\right)$ (Fig. 1).

Tree productivity. Radical pruning $\left(\mathrm{P}_{\mathrm{r}} \cdot \mathrm{W}_{\mathrm{s}} \cdot \mathrm{F}_{\mathrm{s}} \cdot \mathrm{T}_{\mathrm{s}}\right)$ decreased tree yield by $14 \%$ and hand thinning $\left(\mathrm{P}_{\mathrm{s}} \cdot \mathrm{W}_{\mathrm{s}} \cdot \mathrm{F}_{\mathrm{s}} \cdot \mathrm{T}_{\mathrm{n}}\right)$ by $19 \%$, whereas bark mulching $\left(\mathrm{P}_{\mathrm{s}} \cdot \mathrm{W}_{\mathrm{m}} \cdot \mathrm{F}_{\mathrm{s}} \cdot \mathrm{T}_{\mathrm{s}}\right)$ and excess nitrogen fertigation $\left(\mathrm{P}_{\mathrm{s}} \cdot \mathrm{W}_{\mathrm{s}} \cdot \mathrm{F}_{\mathrm{s}} \cdot \mathrm{T}_{\mathrm{n}}\right)$ increased it by $7 \%$ and $11 \%$, respectively, in comparison with control trees (Table 1; Fig. 2; $P<0.05)$. The negative effect of radical pruning or hand thinning on tree yield disappeared when either was applied in a two-factor treatment with bark mulching $\left(\mathrm{P}_{\mathrm{r}} \cdot \mathrm{W}_{\mathrm{m}} \cdot \mathrm{F}_{\mathrm{s}} \cdot \mathrm{T}_{\mathrm{s}} ; \mathrm{P}_{\mathrm{s}} \cdot \mathrm{W}_{\mathrm{m}} \cdot \mathrm{F}_{\mathrm{s}} \cdot \mathrm{T}_{\mathrm{n}}\right)$ or excess nitrogen fertigation $\left(\mathrm{P}_{\mathrm{r}} \cdot \mathrm{W}_{\mathrm{s}} \cdot \mathrm{F}_{\mathrm{x}} \cdot \mathrm{T}_{\mathrm{s}} ; \mathrm{P}_{\mathrm{s}} \cdot \mathrm{W}_{\mathrm{s}} \cdot \mathrm{F}_{\mathrm{x}} \cdot \mathrm{T}_{\mathrm{n}}\right)$ (Fig. 2). Tr. 12 to 16 generally increased yield compared with $\mathrm{Tr} .2$ to $11 . \mathrm{P}_{\mathrm{r}} \cdot \mathrm{W}_{\mathrm{m}} \cdot \mathrm{F}_{\mathrm{x}} \cdot \mathrm{T}_{\mathrm{s}}$ increased yield by $28 \%$ in comparison with standard treatment (Tr. 1), 16\% in comparison with $\mathrm{P}_{\mathrm{r}} \cdot \mathrm{W}_{\mathrm{m}} \cdot \mathrm{F}_{\mathrm{s}} \cdot \mathrm{T}_{\mathrm{s}}(\mathrm{Tr}$. 6), and $24 \%$ in comparison with $\mathrm{P}_{\mathrm{r}} \cdot \mathrm{W}_{\mathrm{s}} \cdot \mathrm{F}_{\mathrm{x}} \cdot \mathrm{T}_{\mathrm{s}}$ (Tr. 7) (Fig. 2). The highest yield was found for $\mathrm{P}_{\mathrm{r}} \cdot \mathrm{W}_{\mathrm{m}} \cdot \mathrm{F}_{\mathrm{x}} \cdot \mathrm{T}_{\mathrm{n}}$ (Tr.16). Removal of hand thinning from this treatment did not significantly affect the yield (Fig. 2). The interaction between season and treatment effect on yield was seldom significant (Table 1).

Fruit quality and storability. Fruits from radically pruned $\left(\mathrm{P}_{\mathrm{r}} \cdot \mathrm{W}_{\mathrm{s}} \cdot \mathrm{F}_{\mathrm{s}} \cdot \mathrm{T}_{\mathrm{s}}\right)$ or handthinned $\left(\mathrm{P}_{\mathrm{s}} \cdot \mathrm{W}_{\mathrm{s}} \cdot \mathrm{F}_{\mathrm{s}} \cdot \mathrm{T}_{\mathrm{n}}\right)$ trees were $16 \%$ and $13 \%$ larger, respectively, than fruits from trees treated by standard management (Table 1; Fig. 3; $P<0.05)$. The combination of these procedures $\left(\mathrm{P}_{\mathrm{r}} \cdot \mathrm{W}_{\mathrm{s}} \cdot \mathrm{F}_{\mathrm{s}} \cdot \mathrm{T}_{\mathrm{n}} ; \mathrm{Tr}\right.$. 8) increased fruit weight by $20 \%$ in comparison with fruits from only radically pruned trees and by $23 \%$ in comparison with fruits from only handthinned trees (Fig. 3). Fruits from barkmulched trees were $13 \%$ larger than fruits from the control trees. $\mathrm{P}_{\mathrm{r}} \cdot \mathrm{W}_{\mathrm{m}} \cdot \mathrm{F}_{\mathrm{s}} \cdot \mathrm{T}_{\mathrm{s}}$ increased fruit weight by $13 \%$ in comparison with radically pruning alone and by $42 \%$ in comparison with bark mulching alone (Fig. 3). Fruits from the three- (Tr. 12 to 15 ) or fourfactor treatment (Tr. 16) were $50 \%$ to $70 \%$ heavier than fruits from control trees. $\mathrm{P}_{\mathrm{r}} \cdot \mathrm{W}_{\mathrm{m}} \cdot \mathrm{F}_{\mathrm{s}} \cdot \mathrm{T}_{\mathrm{n}}$ caused the largest fruits (Fig. 3).

The peel color of 'Katja' apple was significantly affected by season (Table 1 ). Radical pruning $\left(\mathrm{P}_{\mathrm{r}} \cdot \mathrm{W}_{\mathrm{s}} \cdot \mathrm{F}_{\mathrm{s}} \cdot \mathrm{T}_{\mathrm{s}}\right.$ during two seasons) increased red color $\left(25 \%\right.$ lower $\mathrm{H}^{\circ}$ value) in comparison with fruits from control trees (Table 2; $P<0.05$ ). $\mathrm{P}_{\mathrm{r}} \cdot \mathrm{W}_{\mathrm{s}} \cdot \mathrm{F}_{\mathrm{s}} \cdot \mathrm{T}_{\mathrm{n}}$ or $\mathrm{P}_{\mathrm{r}} \cdot \mathrm{W}_{\mathrm{m}} \cdot \mathrm{F}_{\mathrm{s}} \cdot \mathrm{T}_{\mathrm{s}}$ did not improve fruit coloration in comparison with radical pruning alone (Table 2). Excess $\mathrm{N}$ fertigation always had a negative effect on fruit coloration. It also neutralized the positive effect of radical pruning when a combination $\left(\mathrm{P}_{\mathrm{r}} \cdot \mathrm{W}_{\mathrm{s}} \cdot \mathrm{F}_{\mathrm{x}} \cdot \mathrm{T}_{\mathrm{s}}\right)$ 


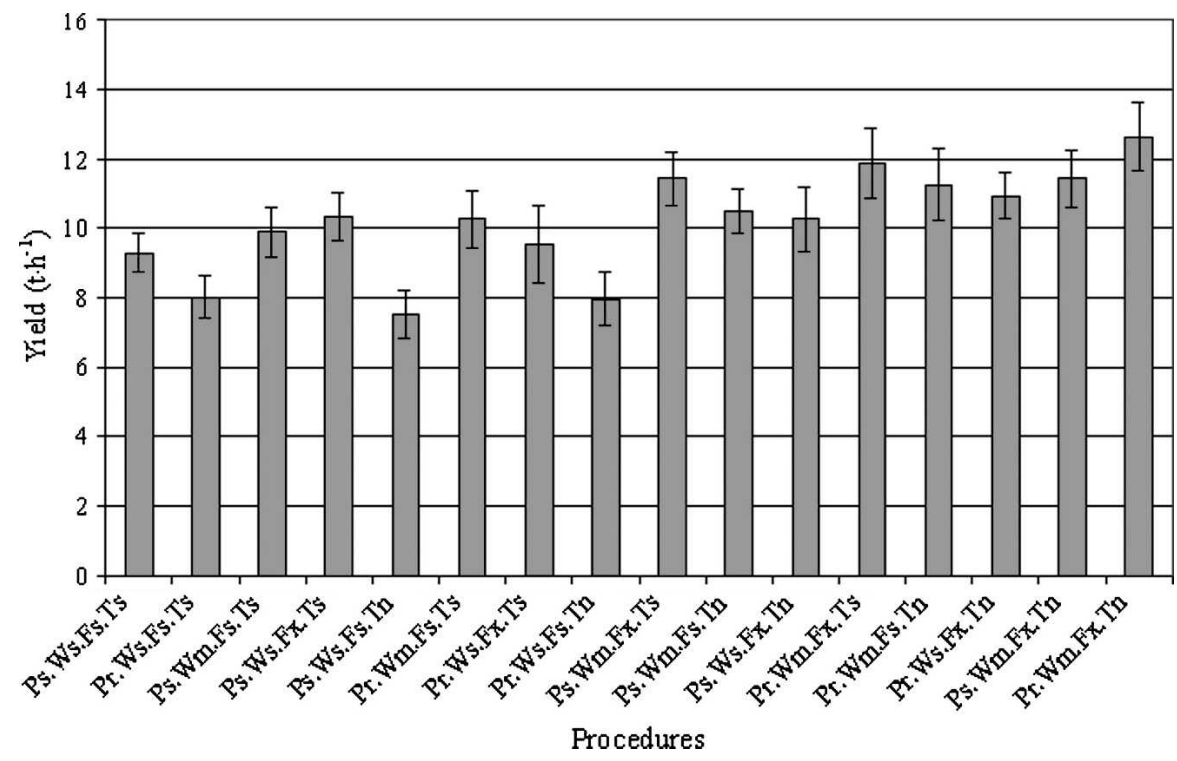

Fig. 2. Tree production of 'Katja' apples (2001 to 2003 average) Bars represent SD \pm . Values are means of 4 years, 16 models of six single blocks $(\mathrm{n}=384)$. Interaction between season and models was not significant at $(P<0.05)$. Procedures: Pruning $(\mathrm{P})$ - standard $\left(\mathrm{P}_{\mathrm{s}}\right)$ or radical $\left(\mathrm{P}_{\mathrm{r}}\right)$; weed control $(\mathrm{W})$ - herbicides $\left(\mathrm{W}_{\mathrm{s}}\right)$ or bark mulching $\left(\mathrm{W}_{\mathrm{m}}\right)$; fertilization $(\mathrm{F})$ - standard fertigation $\left(\mathrm{F}_{\mathrm{s}}\right)$ or excess nitrogen $\left(\mathrm{F}_{\mathrm{x}}\right)$; and thinning $(\mathrm{T})-$ no thinning $\left(\mathrm{T}_{\mathrm{s}}\right)$ or hand thinning $\left(\mathrm{T}_{\mathrm{n}}\right)$.

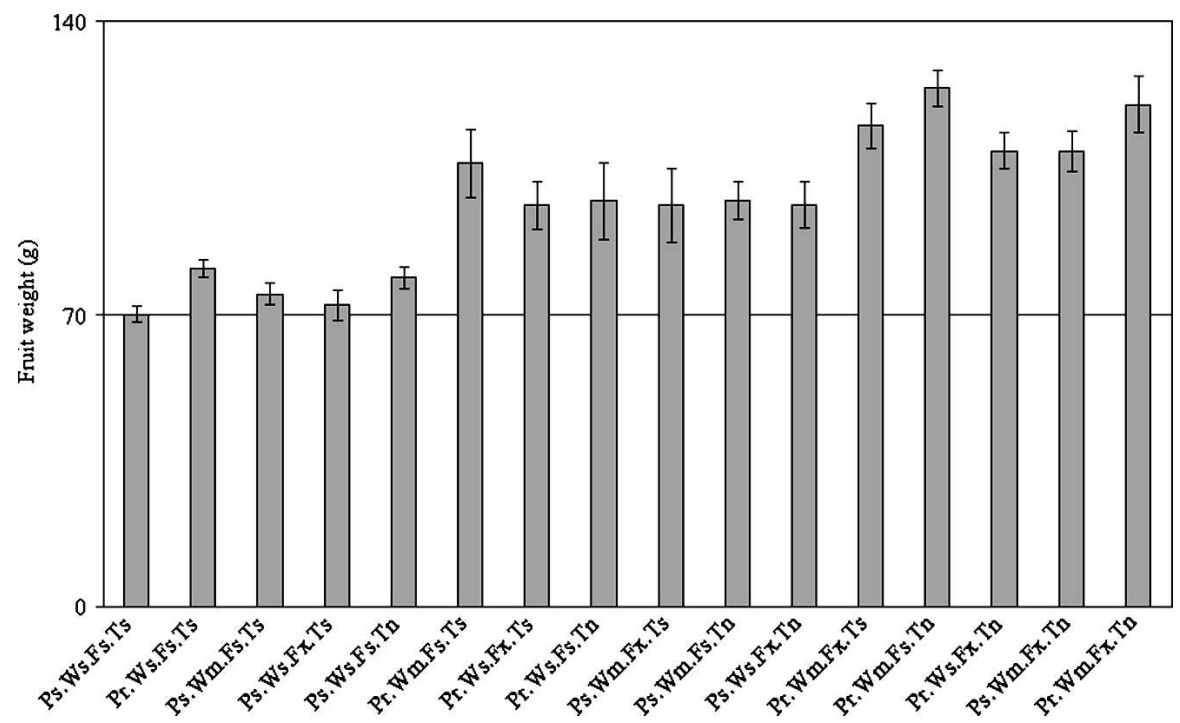

Fig. 3. Mean weight of 'Katja' apples (2001 to 2003 average). Bars represent SD \pm . Values are means of 4 years, 16 models of six single blocks $(n=384)$. Interaction between season and models was not significant at $P<0.05$. Procedures: Pruning $(\mathrm{P})$-standard $\left(\mathrm{P}_{\mathrm{s}}\right)$ or radical $\left(\mathrm{P}_{\mathrm{r}}\right)$; weed control $(\mathrm{W})$ - herbicides $\left(\mathrm{W}_{\mathrm{s}}\right)$ or bark mulching $\left(\mathrm{W}_{\mathrm{m}}\right)$; fertilization $(\mathrm{F})$ - standard fertigation $\left(\mathrm{F}_{\mathrm{s}}\right)$ or excess nitrogen $\left(\mathrm{F}_{\mathrm{x}}\right)$; and thinning $(\mathrm{T})$ - no thinning $\left(\mathrm{T}_{\mathrm{s}}\right)$ or hand thinning $\left(\mathrm{T}_{\mathrm{n}}\right)$.

of these two treatments was applied (Table $2)$. The best fruit coloration during three seasons $\left(40 \%\right.$ lower $\mathrm{H}^{\circ}$ value) was achieved by $\mathrm{P}_{\mathrm{r}} \cdot \mathrm{W}_{\mathrm{m}} \cdot \mathrm{F}_{\mathrm{s}} \cdot \mathrm{T}_{\mathrm{n}}$ (Table 2$)$.

Trees that were treated by $\mathrm{P}_{\mathrm{r}} \cdot \mathrm{W}_{\mathrm{s}} \cdot \mathrm{F}_{\mathrm{s}} \cdot \mathrm{T}_{\mathrm{n}}$ had $15 \%$ firmer fruits than control trees. Applying bark mulching to this two-factor treatment $\left(\mathrm{P}_{\mathrm{r}} \cdot \mathrm{W}_{\mathrm{m}} \cdot \mathrm{F}_{\mathrm{s}} \cdot \mathrm{T}_{\mathrm{n}}\right)$ improved fruit firmness by $20 \%$ (Table $2 ; P<0.05$ ). Treatments did not show any significant effect on fruit sweetness. $\mathrm{P}_{\mathrm{r}} \cdot \mathrm{W}_{\mathrm{m}} \cdot \mathrm{F}_{\mathrm{s}} \cdot \mathrm{T}_{\mathrm{s}}$ or $\mathrm{P}_{\mathrm{s}} \cdot \mathrm{W}_{\mathrm{m}} \cdot \mathrm{F}_{\mathrm{s}} \cdot \mathrm{T}_{\mathrm{n}}$ caused higher fruit acidity in comparison with other treatments (Table 2). Trees treated with a three-factor combination of these pro- loss. Radical pruning $\left(\mathrm{P}_{\mathrm{r}} \cdot \mathrm{W}_{\mathrm{s}} \cdot \mathrm{F}_{\mathrm{s}} \cdot \mathrm{T}_{\mathrm{s}}\right)$ and excess $\mathrm{N}$ fertigation $\left(\mathrm{P}_{\mathrm{s}} \cdot \mathrm{W}_{\mathrm{s}} \cdot \mathrm{F}_{\mathrm{x}} \cdot \mathrm{T}_{\mathrm{s}}\right)$ increased storage decay by $20 \%$ and $11 \%$, respectively (Tables 1 and 3). $\mathrm{P}_{\mathrm{r}} \cdot \mathrm{W}_{\mathrm{m}} \cdot \mathrm{F}_{\mathrm{s}} \cdot \mathrm{T}_{\mathrm{s}}$ or $\mathrm{P}_{\mathrm{r}} \cdot \mathrm{W}_{\mathrm{m}} \cdot \mathrm{F}_{\mathrm{s}} \cdot \mathrm{T}_{\mathrm{n}}$ improved fruit resistance to pathogen rot (54\% and $70 \%$ lower decay, respectively, in comparison with fruits from control trees) (Table 3). Fruits from standard treatment lost $30 \%, 10 \%$, and $50 \%$ of their firmness, SSC, and malic acid content, respectively, during storage (Table 3). $P_{s} \cdot W_{m} \cdot F_{s} \cdot T_{n}$ or $P_{r} \cdot W_{m} \cdot F_{s} \cdot T_{n}$ resulted in a lower decline in fruit firmness $(\approx 20 \%)$ and malic acid content $(\approx 10 \%)$ in comparison with fruits from control or radically pruned trees (Table 3 ). $\mathrm{P}_{\mathrm{s}} \cdot \mathrm{W}_{\mathrm{s}} \cdot \mathrm{F}_{\mathrm{x}} \cdot \mathrm{T}_{\mathrm{s}}$ or $\mathrm{P}_{\mathrm{r}} \cdot \mathrm{W}_{\mathrm{s}} \cdot \mathrm{F}_{\mathrm{x}} \cdot \mathrm{T}_{\mathrm{s}}$ caused faster decrease in fruit firmness and flavor quality $(40 \%)$ in comparison with treatments without excess nitrogen (Table 3).

\section{Discussion}

The potential to change the growth habit of 'Katja' trees (improvement of vegetative growth, regulation of bearing, and production of high fruit quality and storability) by the use of different cultural practices depends on the ability of these practices to adjust the competition for carbohydrate consumption between vegetative and generative organs so that more carbohydrates are translocated to the fruits and not to new vegetative growth (Bepete and Lakso, 1998; Goffinet et al., 1995; Tromp, 2005). Contradictory to earlier results in other apple cultivars (Fathi and Mukhtar, 1998; Ghosh et al., 2004), the present investigation showed that one-factor orchard management treatments (Tr. 2 to 5) were not sufficient to improve the productivity, quality, and storability of 'Katja' apples. Although radical pruning increased TCSA, encouraged shoot growth, and improved fruit coloration, it simultaneously decreased yield and fruit storability, attributable perhaps to high competition for carbohydrates between fruits and rapid-grown shoots during the first $40 \mathrm{~d}$ after full bloom (Grappadelli et al., 1994). Thinning has been reported to positively influence regulated bearing, light transmission, and fruit quality (Fathi and Mukhtar, 1998; Ystaas, 1992), but such a relationship was not found for 'Katja' apples in the present investigation, where hand thinning decreased yield without any color improvement. Similarly to previous results (Tahir et al., 2005), bark mulching increased yield and fruit firmness, but in the present investigation, it did not improve vegetative growth as has been reported by several authors (Choi-Seong and Choi, 2000; Pfammatter and Dossimoz, 1997). High nitrogen rates caused better growth and yield, but also decreased light transmission, promoted greenness, and decreased red color, as has been reported previously (Meheriuk et al., 1996). High nitrogen negatively affected fruit storage potential as a result of increasing shading and decreasing fruit $\mathrm{Ca}$ content.

Interactions between the management procedures were investigated in 10 different treatments (Tr. 6 to 16). A combination of 
Table 3. Fruit mineral content at harvest and fruit quality of Katja apple after storage at $2.5^{\circ} \mathrm{C}$ and $90 \%$ relative humidity for 10 weeks (2000 to 2003 average). ${ }^{z}$

\begin{tabular}{|c|c|c|c|c|c|c|c|}
\hline \multirow[b]{3}{*}{ Tr. } & \multirow[b]{3}{*}{ Procedures } & \multirow{3}{*}{$\begin{array}{c}\text { Decay } \\
(\%)\end{array}$} & \multirow{3}{*}{$\begin{array}{c}\text { Firmness } \\
(\mathrm{N})\end{array}$} & \multirow{3}{*}{$\begin{array}{l}\mathrm{SSC}^{\mathrm{y}} \\
(\%)\end{array}$} & \multirow{3}{*}{$\begin{array}{l}\text { Malic acid } \\
\left(\mathrm{mg} / 100 \mathrm{~g}^{-1}\right. \\
\mathrm{FW})^{\mathrm{x}}\end{array}$} & \multicolumn{2}{|c|}{ Mineral content } \\
\hline & & & & & & $\mathrm{N}$ & $\mathrm{K} / \mathrm{Ca}$ \\
\hline & & & & & & \multicolumn{2}{|c|}{$\left(\mathrm{mg} / 100 \mathrm{~g}^{-1} \mathrm{FW}\right)$} \\
\hline 1 & $\mathrm{P}_{\mathrm{s}} \cdot \mathrm{W}_{\mathrm{s}} \cdot \mathrm{F}_{\mathrm{s}} \cdot \mathrm{T}_{\mathrm{s}}$ & $7.2 \mathrm{ab}$ & $48.0 \mathrm{c}$ & $10.5 \mathrm{~g}$ & $337.0 \mathrm{~g}$ & $38.0 \mathrm{ab}$ & $41.2 \mathrm{ab}$ \\
\hline 2 & $\mathrm{P}_{\mathrm{r}} \cdot \mathrm{W}_{\mathrm{s}} \cdot \mathrm{F}_{\mathrm{s}} \cdot \mathrm{T}_{\mathrm{s}}$ & $8.7 \mathrm{a}$ & $49.0 \mathrm{c}$ & $10.8 \mathrm{~g}$ & $399.1 \mathrm{efg}$ & $39.0 \mathrm{ab}$ & $43.7 \mathrm{ab}$ \\
\hline 3 & $\mathrm{P}_{\mathrm{s}} \cdot \mathrm{W}_{\mathrm{m}} \cdot \mathrm{F}_{\mathrm{s}} \cdot \mathrm{T}_{\mathrm{s}}$ & $5.1 \mathrm{abc}$ & $54.9 \mathrm{abc}$ & $11.9 \mathrm{bcdef}$ & $587.3 \mathrm{~b}$ & $40.0 \mathrm{ab}$ & $33.9 \mathrm{ab}$ \\
\hline 4 & $\mathrm{P}_{\mathrm{s}} \cdot \mathrm{W}_{\mathrm{s}} \cdot \mathrm{F}_{\mathrm{x}} \cdot \mathrm{T}_{\mathrm{s}}$ & $8.0 \mathrm{a}$ & $49.0 \mathrm{c}$ & $11.1 \mathrm{efg}$ & $362.9 \mathrm{fg}$ & $43.0 \mathrm{ab}$ & $38.3 \mathrm{ab}$ \\
\hline 5 & $\mathrm{P}_{\mathrm{s}} \cdot \mathrm{W}_{\mathrm{s}} \cdot \mathrm{F}_{\mathrm{s}} \cdot \mathrm{T}_{\mathrm{n}}$ & $6.6 \mathrm{ab}$ & $53.9 \mathrm{abc}$ & $12.2 \mathrm{abcd}$ & 478.8 bcde & $28.0 \mathrm{~b}$ & $52.9 \mathrm{a}$ \\
\hline 6 & $\mathrm{P}_{\mathrm{r}} \cdot \mathrm{W}_{\mathrm{m}} \cdot \mathrm{F}_{\mathrm{s}} \cdot \mathrm{T}_{\mathrm{s}}$ & $3.3 \mathrm{c}$ & $51.0 \mathrm{abc}$ & $12.5 \mathrm{ab}$ & $564.8 \mathrm{bc}$ & $33.3 \mathrm{ab}$ & $37.6 \mathrm{ab}$ \\
\hline 7 & $\mathrm{P}_{\mathrm{r}} \cdot \mathrm{W}_{\mathrm{s}} \cdot \mathrm{F}_{\mathrm{x}} \cdot \mathrm{T}_{\mathrm{s}}$ & $8.2 \mathrm{a}$ & $50.0 \mathrm{bc}$ & $11.1 \mathrm{defg}$ & 416.1 defg & $41.7 \mathrm{ab}$ & $37.0 \mathrm{ab}$ \\
\hline 8 & $\mathrm{P}_{\mathrm{r}} \cdot \mathrm{W}_{\mathrm{s}} \cdot \mathrm{F}_{\mathrm{s}} \cdot \mathrm{T}_{\mathrm{n}}$ & $3.5 \mathrm{bc}$ & $54.9 \mathrm{abc}$ & $11.9 \mathrm{bcdef}$ & $459.9 \mathrm{cdef}$ & $37.7 \mathrm{ab}$ & $36.7 \mathrm{ab}$ \\
\hline 9 & $\mathrm{P}_{\mathrm{s}} \cdot \mathrm{W}_{\mathrm{m}} \cdot \mathrm{F}_{\mathrm{x}} \cdot \mathrm{T}_{\mathrm{s}}$ & $6.4 \mathrm{ab}$ & $51.0 \mathrm{abc}$ & $11.9 \mathrm{bcdef}$ & 487.2 bcde & $44.3 \mathrm{a}$ & $36.7 \mathrm{ab}$ \\
\hline 10 & $\mathrm{P}_{\mathrm{s}} \cdot \mathrm{W}_{\mathrm{m}} \cdot \mathrm{F}_{\mathrm{s}} \cdot \mathrm{T}_{\mathrm{n}}$ & $3.8 \mathrm{bc}$ & $61.7 \mathrm{a}$ & $13.1 \mathrm{a}$ & $698.9 \mathrm{a}$ & $42.1 \mathrm{ab}$ & $38.1 \mathrm{ab}$ \\
\hline 11 & $\mathrm{P}_{\mathrm{s}} \cdot \mathrm{W}_{\mathrm{s}} \cdot \mathrm{F}_{\mathrm{x}} \cdot \mathrm{T}_{\mathrm{n}}$ & $5.1 \mathrm{abc}$ & $52.9 \mathrm{abc}$ & $10.8 \mathrm{~g}$ & $322.4 \mathrm{~g}$ & $45.2 \mathrm{a}$ & $42.7 \mathrm{ab}$ \\
\hline 12 & $\mathrm{P}_{\mathrm{r}} \cdot \mathrm{W}_{\mathrm{m}} \cdot \mathrm{F}_{\mathrm{x}} \cdot \mathrm{T}_{\mathrm{s}}$ & $7.8 \mathrm{a}$ & $50.0 \mathrm{abc}$ & 11.2 cdefg & 482.3 bcde & $42.0 \mathrm{ab}$ & $39.3 \mathrm{ab}$ \\
\hline 13 & $\mathrm{P}_{\mathrm{r}} \cdot \mathrm{W}_{\mathrm{m}} \cdot \mathrm{F}_{\mathrm{s}} \cdot \mathrm{T}_{\mathrm{n}}$ & $2.1 \mathrm{c}$ & $61.7 \mathrm{a}$ & $12.2 \mathrm{abc}$ & $516.8 \mathrm{bcd}$ & $39.0 \mathrm{ab}$ & $34.1 \mathrm{ab}$ \\
\hline 14 & $\mathrm{P}_{\mathrm{r}} \cdot \mathrm{W}_{\mathrm{s}} \cdot \mathrm{F}_{\mathrm{x}} \cdot \mathrm{T}_{\mathrm{n}}$ & $5.6 \mathrm{abc}$ & $49.0 \mathrm{c}$ & $10.9 \mathrm{~g}$ & $366.2 \mathrm{fg}$ & $38.0 \mathrm{ab}$ & $39.4 \mathrm{ab}$ \\
\hline 15 & $\mathrm{P}_{\mathrm{s}} \cdot \mathrm{W}_{\mathrm{m}} \cdot \mathrm{F}_{\mathrm{x}} \cdot \mathrm{T}_{\mathrm{n}}$ & $3.8 \mathrm{bc}$ & $51.9 \mathrm{abc}$ & $11.2 \mathrm{defg}$ & 468.2 cdef & $46.0 \mathrm{a}$ & $32.9 \mathrm{~b}$ \\
\hline 16 & $\mathrm{P}_{\mathrm{r}} \cdot \mathrm{W}_{\mathrm{m}} \cdot \mathrm{F}_{\mathrm{x}} \cdot \mathrm{T}_{\mathrm{n}}$ & $5.2 \mathrm{abc}$ & $51.9 \mathrm{abc}$ & $12.4 \mathrm{ab}$ & 485.2 bcde & $43.0 \mathrm{ab}$ & $32.9 \mathrm{~b}$ \\
\hline
\end{tabular}

Means in the same column followed by same letter did not differ significantly at $P<0.05$ (Tukey's studentized range - honestly significant difference).

${ }^{2}$ Pruning $(\mathrm{P})$ - standard $\left(\mathrm{P}_{\mathrm{s}}\right)$ or radical $\left(\mathrm{P}_{\mathrm{r}}\right)$; weed control $(\mathrm{W})$-herbicides $\left(\mathrm{W}_{\mathrm{s}}\right)$ or bark mulching $\left(\mathrm{W}_{\mathrm{m}}\right)$; fertilization $(\mathrm{F})$ - standard fertigation $\left(\mathrm{F}_{\mathrm{s}}\right)$ or excess nitrogen $\left(\mathrm{F}_{\mathrm{x}}\right)$; and thinning $(\mathrm{T})$ - no thinning $\left(\mathrm{T}_{\mathrm{s}}\right)$ or hand thinning $\left(\mathrm{T}_{\mathrm{n}}\right)$.

${ }^{\mathrm{y}}$ Soluble solid concentration.

${ }^{\mathrm{x}}$ Fresh weight.

radical pruning, which improved red color as a result of better light distribution in the canopy (Marini and Barden, 2004), and excess nitrogen fertigation, which increased yield, was not a useful solution because this combination also led to high storage decay and insufficient fruit color. Radical pruning in combination with bark mulching and hand thinning decreased the negative effect of each practice alone. Except for the first year, this combination caused the highest relative change in TCSA, larger fruits, high regular yield, and better fruit quality (color and flavor) and storability. This indicates that applying these procedures together could activate or neutralize some of the positive or negative effects originating from each of them alone. Early decapitation of the currentyear shoots might provide sufficient carbohydrates for the formation of high-quality buds, resulting in large and healthy fruits. An optimum early season hand thinning ( 1 to 2 weeks after full bloom) not only led to improved fruit size, regulated bearing, and decreased storage decay, but also improved the pruning effect on light penetration within the crown. Bark mulching consolidates fruit quality and storability probably by saving soil moisture, increasing nutrition availability, and adjusting $\mathrm{K} / \mathrm{Ca}$ ratio (Tahir et al., 2005). Bark mulching in $\mathrm{P}_{\mathrm{r}} \cdot \mathrm{W}_{\mathrm{m}} \cdot \mathrm{F}_{\mathrm{s}} \cdot \mathrm{T}_{\mathrm{n}}$ cancelled the negative effect of thinning and radical pruning on $\mathrm{Ca}$ content, resulting in better storage potential. Because of the nonsignificant difference between this treatment and the four-factor treatment (i.e., with excess fertigation also included), growers can apply this threefold treatment to limit excess application of nitrogen and thereby decrease their costs and protect the environment.

The positive interacting effects of radical pruning, bark mulching, and hand thinning were very clearly demonstrated in this study and thus provide a method to solve production problems for 'Katja' apple orchards.

\section{Literature Cited}

Basak, A. 1999. The storage quality of apples after fruitlets thinning. Acta Hort. 485:47-54.

Bepete, M. and A.N. Lakso. 1998. Differential effects of shade on early season fruit and shoot growth rates in 'Empire' apple branches. HortScience 33:823-825.

Bergh, O. 1990. Effect of time of hand thinning on apple fruit size. S. Afr. J. Plant Soil 7: $1-10$.

Choi-Seong, Y. and S. Choi. 2000. The effect of mulching material on the shoot and root growth and fruit quality of 'Fugi'/M.26 apple. J. Korean Soc. HortScience 41:512-516.

Fathi, M.A. and H. Mukhtar. 1998. Influence of summer pruning on growth, fruit set and fruit quality of Anna apple trees. Egypt. J. Agr. Res. 76:721-732.

Fausett, J.B. and C.R. Rom. 2001. The effects of transitioning a mature high-density orchard from standard herbicide ground cover management system to organic ground-cover management systems. Arkansas Agr. Expt. Sta. 483: 33-36.

Fernandez, G.C.J. 2007. Design and analysis of commonly used comparative horticultural experiments. HortScience 42:1052-1069.

Ghosh, S.N., S. Manna, and B. Mathew. 2004 Effect of nitrogen and potassium fertilization on custard apple grown under rain fed late rite soils. Environ. Ecol. 22:144-147.

Goffinet, M.C., T.L. Robinson, and A.N. Lakso. 1995. A comparison of 'Empire' apple fruit in unthinned and hand-thinned trees. J. Hort. Sci. 70:375-387.

Goldschmidt, R.E. 1997. Regulating trees of apple and pear by pruning and bending. Swedish J. Agr. Res. 27:45-52.

Grappadelli, L.C., A.N. Lakso, and J.A. Flore. 1994. Early season pattern of carbohydrate portioning in exposed and shaded apple branches. J. Amer. Soc. Hort. Sci. 119:596603.

Jadczuk, E., A. Pietranek, and R. Dziuban. 2001. Irrigation and $\mathrm{K}$ fertilization effects on the cropping and fruit quality of 'Katja' apple trees on four different rootstocks. Folia. Hort. 13: 47-60 (abstr.).

Krzewinska, D., A. Basak, A. Mika, and B. Michalska. 2002. The effect of time and intensity of hand thinning on the distribution of apples in size and colour classes. Zeszyty Naukowe Instytutu Sadownictwa i Kwiaciarstwa W Skierniewicach. 10:89-99 (abstr.).

Link, H. 2000. Significance of flower and fruit thinning on fruit quality. Plant Growth Regulat. 31:17-26.

Marini, R.P. 2004. Combinations of ethophon and Accel for thinning 'Delicious' apple trees. J. Amer. Soc. Hort. Sci. 129:175-181.

Marini, R.P. and J.A. Barden. 2004. Yield, fruit size, red colour, and a hand economic analysis for 'Delicious' and 'Empire' in the NC-140 1994 System Trail in Virginia. J. Amer. Pomol. Soc. 58:4-11.

McGuire, R.G. 1992. Reporting of objective colour measurements. HortScience 27:1254 1255.

Meheriuk, M., D.L. Mckenzie, G.H. Neilsen, and J.W. Hall. 1996. Fruit pigmentation of four green apple cultivars responds to urea sprays but not to nitrogen fertilization. HortScience 31:992-993

Pfammatter, W. and A. Dossimoz. 1997. Influence of irrigation and ground cover on development and yields of young apple trees. Revue. Suisse. De. Viticulture. D'Arboriculture et d'Horticulture 29:301-304.

Pretorious, J.J.B., S.J.E. Wand, and K.L. Theron. 2004. Fruit and shoot growth following combined girdling and thinning of 'Royal Gala' apple trees. Acta Hort. 636:401-407.

Rein, A. 1996. Apple storage disorders (original title in Swedish: Skador på äpplen vid inlagring). Swedish Board of Agr. Agro. Info. 3810 Gvarv.

Rubauskis, E., M. Skrivele, I. Dimza, and V. Berlands. 2002. The response of apple trees to fertigation and mulch. Sodininkyste ir Darzininkyste. 21:126-133.

Tahir, I., E. Johansson, and M.E. Olsson. 2005. Groundcover materials improve quality and storability of 'Aroma' apples. HortScience 40: 1416-1420.

Tahir, I., E. Johansson, and M.E. Olsson. 2007. Improvement of quality and storability of apple cv. Aroma by adjustment of some pre-harvest conditions. Scientia Hort. 12:164-171.

Tomala, K. 1997. Orchard factors affecting nutrient content and fruit quality. Acta Hort. 448: 257-264.

Tromp, J. 2005. Mineral nutrition, p. 55-63. In: Tromp, J., A.D. Webster, and S.J. Wertheim (eds.). Fundamentals of temperate zone tree fruit production. Backhuys Publishers, Leiden, The Netherlands.

Wertheim, S.J. 2005. Pruning, p. 176-185. In: Tromp, J., A.D. Webster, and S.J. Wertheim (eds.). Fundamentals of temperate zone tree fruit production. Backhuys Publishers, Leiden, The Netherlands.

Wójcik, P., K. Rutkoeski, and W. Treder. 2001. Quality and storability of 'Gala' apples as affected by crop load. Folia Hort. 13:89-96 (abstr.).

Ystaas, J. 1992. Effects of summer pruning on yield, fruit size, and fruit quality of the apple cultivar 'Summerred'. Acta Hort. 322:277282. 